\title{
A Numerical Study of One-Dimensional Hyperbolic Telegraph Equation
}

\author{
Shaheed N. Huseen \\ Thi-Qar University, Faculty of Computer Science and Mathematics, Mathematics Department, Thi-Qar, Iraq.
}

Received: October 13, 2016 / Accepted: November 10, 2016 / Published: February 25, 2017.

\begin{abstract}
In this paper, an approximate solution for the one-dimensional hyperbolic telegraph equation by using the q-homotopy analysis method (q-HAM) is proposed.The results shows that the convergence of the q- homotopy analysis method is more accurate than the convergence of the homotopy analysis method (HAM).
\end{abstract}

Key words: q-Homotopy analysis method, one-dimensional hyperbolic telegraph equation.

\section{Introduction}

The second-order linear hyperbolic telegraph equation in one-space dimension has the form:

$$
\begin{gathered}
u_{t t}+2 \alpha u_{t}+\beta^{2} u=u_{x x}+f(x, t), \\
a \leq x \leq b, t \geq 0
\end{gathered}
$$

subject to initial conditions

$$
\begin{aligned}
& u(x, 0)=f_{1}(x), a \leq x \leq b \\
& u_{t}(x, 0)=f_{2}(x), a \leq x \leq b
\end{aligned}
$$

and Dirichlet boundary conditions

$$
u(a, t)=g_{1}(t), u(b, t)=g_{2}(t),
$$

where $\alpha$ and $\beta$ are known constant coefficients.

For $>0, \beta=0$ Eq. (1) represents a damped wave equation and for $\alpha>0, \beta>0$, it is called telegraph equation.

Equations of the form Eq. (1) arise in the study of propagation of electrical signals in a cable of transmission line and wave phenomena. Interaction between convection and diffusion or reciprocal action of reaction and diffusion describes a number of nonlinear phenomena in physical, chemical and biological process $[17,18,34,36]$. In fact the telegraph equation is more suitable than ordinary diffusion

Corresponding author: Shaheed N. Huseen, Thi-Qar University, Faculty of Computer Science and Mathematics, Mathematics Department, Thi-Qar, Iraq. E-mail: shn_n2002@yahoo.com equation in modeling reaction diffusion for such branches of sciences. For example biologists encounter these equations in the study of pulsate blood flow in arteries and in one- dimensional random motion of bugs along a hedge [33]. Also the propagation of acoustic waves in Darcy-type porous media [35], and parallel flows of viscous Maxwell fluids [1] are just some of the phenomena governed [8,19] by Eq.(1). In [2], a numerical scheme for solving the secondorder one-space-dimensional linear hyperbolic equation has been presented by using the shifted Chebyshev cardinal functions. Dehghan and Shokri $[3,4]$ have studied a numerical scheme to solve one and two-dimensional hyperbolic equations using collocation points and the thin-plate-spline radial basis functions. In [34], a numerical method, based on the combination of a high-order compact finite-difference scheme was used to approximate the spatial derivative and the collocation technique for the time component was proposed to solve the one-space-dimensional linear hyperbolic equation. Dehghan and Mohebbi [5] have developed an efficient approach for solving the two dimensional linear hyperbolic telegraph equation, using the compact finite difference approximation of fourth order and collocation method. A numerical scheme, based on the shifted Chebyshev tau method 
was proposed in [37] to solve this equation. In [9], an explicit difference scheme has been discussed for the numerical solution of the linear hyperbolic equation of the form Eq. (1).

The standard homotopy analysis method (HAM) is an analytic method that provides series solutions for nonlinear partial differential equations and has been firstly proposed by Liao 1992. Liao [20-31] developed and applied the homotopy analysis method(HAM) to deal with a lot of nonlinear problems. The HAM provides a simple way to ensure the convergence of a solution in a series form under certain conditions. The Homotopy Analysis Method (HAM) is based on homotopy, a fundamental concept in topology. Briefly, in HAM, one constructs a continuous mapping of an initial guess approximation to the exact solution of the problems to be considered. An auxiliary linear operator is chosen to construct such kind of continuous mapping and an auxiliary parameter is used to ensure convergence of the solution series. The method enjoys great freedom in choosing initial approximation and auxiliary linear operator. In 2004, Liao published the book [32] in which he summarized the basic ideas of the homotopy analysis method and gave the details of his approach both in the theory and on a large number of practical examples.

El-Tawil and Huseen [6] proposed a method namely q-homotopy analysis method (q-HAM) which is a more general method of HAM. The essential idea of this method is to introduce a homotopy parameter, say $q$, which varies from 0 to $1 / n$ ,$n \geq 1$ and a nonzero auxiliary parameter $h$. At $q=0$, the system of equations usually has been reduced to a simplified form which normally admits a rather simple solution. As $q$ gradually increases continuously toward $1 / n$, the system goes through a sequence of deformations, and the solution at each stage is close to that at the previous stage of the deformation. Eventually at $q=1 / n$, the system takes the original form of the equation and the final stage of the deformation gives the desired solution. The q-HAM has been successfully applied to numerous problems in science and engineering $[6,7$, 11-16].

\section{2. q-Homotopy Analysis Method (q-HAM)}

Consider the following differential equation:

$$
N[u(x, t)]-f(x, t)=0
$$

where $N$ is a nonlinear operator, $(x, t)$ denotes independent variables, $f(x, t)$ is a known function and $u(x, t)$ is an unknown function.

Let us construct the so-called zero-order deformation equation:

$$
\begin{aligned}
& (1-n q) L\left[\varnothing(x, t ; q)-u_{0}(x, t)\right]= \\
& q h H(x, t)(N[\varnothing(x, t ; q)]-f(x, t))
\end{aligned}
$$

Where $n \geq 1, q \in\left[0, \frac{1}{n}\right]$ denotes the so-called embedded parameter, $L$ is an auxiliary linear operator with the property $L[f]=0$ when $f=0, h \neq 0$ is an auxiliary parameter, $H(x, t)$ denotes a non-zero auxiliary function.

It is obvious that when $q=0$ and $q=\frac{1}{n}$ equation (2) becomes:

$$
\emptyset(x, t ; 0)=u_{0}(x, t), \varnothing\left(x, t ; \frac{1}{n}\right)=u(x, t)
$$

respectively. Thus as $q$ increases from 0 to $\frac{1}{n}$, the solution $\varnothing(x, t ; q)$ varies from the initial guess $u_{0}(x, t)$ to the solution $u(x, t)$. Having the freedom to choose $u_{0}(x, t), L, h, H(x, t)$, we can assume that all of them can be properly chosen so that the solution $\emptyset(x, t ; q)$ of equation (3) exists for $q \in\left[0, \frac{1}{n}\right]$

Expanding $\emptyset(x, t ; q)$ in Taylor series, one has:

$$
\emptyset(x, t ; q)=u_{0}(x, t)+\sum_{m=1}^{+\infty} u_{m}(x, t) q^{m}
$$

Where:

$$
u_{m}(x, t)=\frac{1}{m !}{\frac{\partial^{m} \emptyset(x, t ; q)}{\partial q^{m}}}_{q=0}
$$

Assume that $h, H(x, t), u_{0}(x, t), L$ are so properly 
chosen such that the series (5) converges at $q=\frac{1}{n}$ and:

$$
\begin{gathered}
u(x, t)=\emptyset\left(x, t ; \frac{1}{n}\right)= \\
u_{0}(x, t)+\sum_{m=1}^{+\infty} u_{m}(x, t)\left(\frac{1}{n}\right)^{m}
\end{gathered}
$$

Defining the vector $u_{r}(x, t)=\left\{u_{0}(x, t), u_{1}(x, t), u_{2}(x, t), \ldots, u_{r}(x, t)\right\}$, differentiating equation (3) $m$ times with respect to $q$ and then setting $q=0$ and finally dividing them by $m$ ! we have the so-called $m^{\text {th }}$ order deformation equation:

$$
\begin{gathered}
L\left[u_{m}(x, t)-k_{m} u_{m-1}(x, t)\right]= \\
h H(x, t) R_{m}\left(\overrightarrow{u_{m-1}}(x, t)\right),
\end{gathered}
$$

where:

$$
\begin{gathered}
R_{m}\left(\overrightarrow{u_{m-1}}(x, t)\right) \\
\frac{1}{(m-1) !} \frac{\partial^{m-1}(N[\varnothing(x, t ; q)]-f(x, t))}{\partial q^{m-1}} \\
q=0
\end{gathered}
$$

and:

$$
k_{m}=\left\{\begin{array}{c}
0 m \leq 1 \\
n \text { otherwise }
\end{array}\right.
$$

It should be emphasized that $u_{m}(x, t)$ for $m \geq 1$ is governed by the linear equation (8) with linear boundary conditions that come from the original problem. Due to the existence of the factor $\left(\frac{1}{n}\right)^{m}$, more chances for convergence may occur or even much faster convergence can be obtained better than the standard HAM. It should be noted that the case of $n=1$ in equqtion (2), standard HAM can be reached.

\section{Numerical Examples}

Example 3.1: The second-order hyperbolic telegraph equation of the form Eq. (1) with $\alpha=$ $4, \beta=2$ and $f(x, t)=\left(2-2 \alpha+\beta^{2}\right) \sin (x) e^{-t}$ take the form:

$$
u_{t t}+8 u_{t}+4 u=u_{x x}-2 \sin (x) e^{-t}
$$

The initial conditions are given by

$$
\begin{gathered}
u(x, 0)=\sin (x), u_{t}(x, 0)=-\sin (x), 0 \leq x \\
\leq 2 \pi, t \geq 0
\end{gathered}
$$

The exact solution by $[3,37]$ is

$$
u(x, t)=\sin (x) e^{-t}
$$

This problem was solved by HAM in [10]. For qHAM solution we choose the linear operator:

$$
L[\varnothing(x, t ; q)]=\frac{\partial^{2} \emptyset(x, t ; q)}{\partial t^{2}}
$$

with the property $L\left[c_{1}+c_{2} t\right]=0$, where $c_{1}, c_{2}$ is constants. Using initial approximation $u_{0}(x, t)=\sin (x)(1-t)$, we define a nonlinear operator as

$$
\begin{aligned}
N[\emptyset(x, t ; q)] & =\frac{\partial^{2} \emptyset(x, t ; q)}{\partial t^{2}}+8 \frac{\partial \emptyset(x, t ; q)}{\partial t} \\
+ & 4 \varnothing(x, t ; q)-\frac{\partial^{2} \emptyset(x, t ; q)}{\partial x^{2}}
\end{aligned}
$$

We construct the zero order deformation equation:

$$
\begin{array}{r}
(1-n q) L\left[\varnothing(x, t ; q)-u_{0}(x, t)\right] \\
=q h H(x, t) N[\varnothing(x, t ; q)] .
\end{array}
$$

we can take $H(x, t)=1$, and the $m^{\text {th }}$ order deformation equation is:

$$
L\left[u_{m}(x, t)-k_{m} u_{m-1}(x, t)\right]=h R_{m}\left(\overrightarrow{u_{m-1}}(x, t)\right)
$$

with the initial conditions for $m \geq 1$

$$
u_{m}(x, 0)=0 \text {, }
$$

where $k_{m}$ as define by (10) and

$$
\begin{aligned}
& R_{m}\left(\vec{u}_{m-1}(x, t)\right)=\frac{\partial^{2} u_{m-1}(x, t)}{\partial t^{2}}+8 \frac{\partial u_{m-1}(x, t)}{\partial t} \\
&+4 u_{m-1}(x, t)-\frac{\partial^{2} u_{m-1}(x, t)}{\partial x^{2}}+ \\
& 2\left(1-\frac{1}{n} k_{m}\right) \sin (x) e^{-t}
\end{aligned}
$$

Now the solution of equation (11) for $m \geq 1$ becomes

$$
\begin{aligned}
u_{m}(x, t)=k_{m} u_{m-1}(x, t) & \\
& +h \int_{0}^{t} \int_{0}^{s} R_{m}\left(\overrightarrow{u_{m-1}}(x, r)\right) d r d s+c_{1} \\
& +c_{2} t
\end{aligned}
$$

where the constants of integration $c_{1}$ and $c_{2}$ are determined by the initial conditions (15). 
We can obtain components of the solution using qHAM as follows:

$$
\begin{aligned}
u_{1}(x, t)=- & \frac{1}{6} h\left(12-12 e^{-t}+t(-12+t(9\right. \\
& +5 t))) \operatorname{Sin}[x] \\
u_{2}(x, t)=- & \frac{1}{6} h n\left(12-12 e^{-t}+t(-12+t(9\right. \\
& +5 t))) \operatorname{Sin}[x]-\frac{1}{24} h^{2}(-96 \\
& +96 e^{-t}+t(96+t(-36+t(76 \\
& +5 t(11+t))))) \operatorname{Sin}[x] \\
1 & e^{-t} h^{2}\left(4 2 n \left(-96+e^{t}(96-t(96\right.\right. \\
& +t(-36+t(76+5 t(11+t)))))) \\
& +h\left(8064-e^{t}(8064+t(-8064\right. \\
& +t(4536+t(2520+t(8064 \\
& +t(4704+5 t(133 \\
& +5 t))))))))) \operatorname{Sin}[x]+n\left(-\frac{1}{6} h n(12\right. \\
& -12 e^{-t}+t(-12+t(9 \\
& +5 t))) \operatorname{Sin}[x]-\frac{1}{24} h^{2}(-96 \\
& +96 e^{-t}+t(96+t(-36+t(76 \\
& +5 t(11+t))))) \operatorname{Sin}[x]) \\
& +5,6, t)
\end{aligned}
$$

$u_{m}(x, t),(m=4,5,6, \ldots)$ can be calculated similarly. Then the series solution expression by q- HAM can be written in the form:

$$
\begin{gathered}
u(x, t ; n ; h) \cong U_{M}(x, t ; n ; h)= \\
\sum_{i=0}^{M} u_{i}(x, t ; n ; h)\left(\frac{1}{n}\right)^{i}
\end{gathered}
$$

Equation (16) is an approximate solution to the problem (11) in terms of the convergence parameters $h$ and $n$. To find the valid region of $h$, the $h$-curves given by the $15^{\text {th }}$ order q-HAM approximation at $(x=0.5, t=1)$ and different values of $n$ are drawn in figures $(1-4)$. These figures show the interval of $h$ at which the value of $U_{15}(x, t ; n)$ is constant at certain values of , $t$ and $n$. We choose the horizontal line parallel to $x-\operatorname{axis}(h)$ as a valid region which provides us with a simple way to adjust and control the convergence region of the series solution (16). From these figures, the region of $h$ for the values of $x, t$ and $n$ in the curves becomes larger as $n$ increase. Figure (5) shows the comparison between $U_{15}$ of HAM and $U_{15}$ of q-HAM using different values of $n$ with the exact solution (12), which indicates that the speed of convergence for q-HAM with $n>1$ is faster in comparison with $n=1$.

The absolute errors of the $15^{\text {th }}$ order solutions q-HAM approximate at $x=1$ using different values of $n>1$ compared with $15^{\text {th }}$ order solutions HAM approximate at $x=1$ are calculated by the formula

$$
\text { Absolute Error }=\left|u_{\text {exact }}-u_{\text {apprax }}\right|
$$

Figures $(6-8)$ show that the series solutions obtained by q-HAM at $n>1$ converge faster than $n=1$ (HAM).

Example 3.2: The second-order hyperbolic telegraph equation of the form Eq. (1) with $\alpha=$ $6, \beta=2 \quad$ and $\quad f(x, t)=-2 \alpha \sin (x) \sin (t)+$ $\beta^{2} \cos (\mathrm{t}) \sin (x)$ take the form:

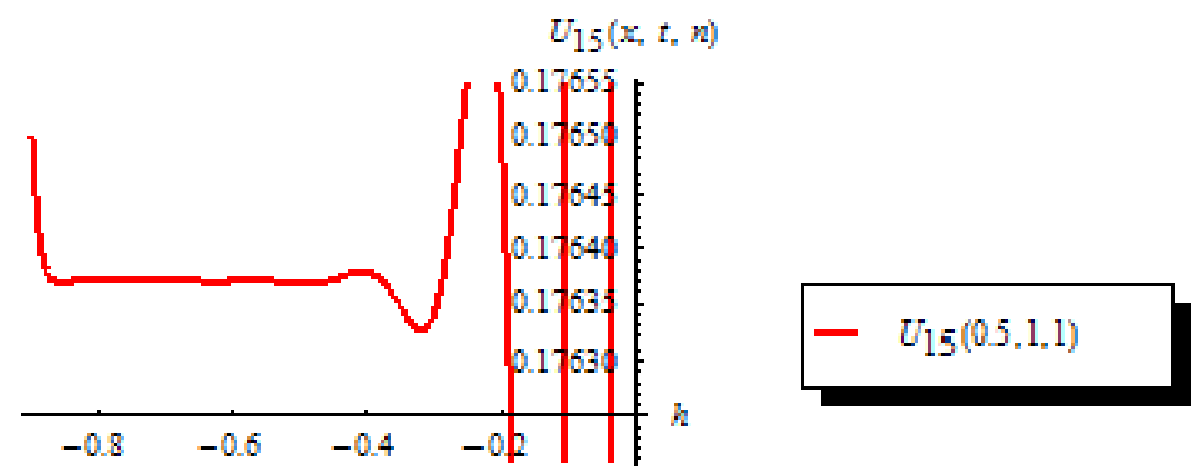

Fig. (1) $h$ - curve for the HAM (q-HAM; $n=1)$ approximation solution, $U_{15}(0.5,1 ; 1)$ of problem (11). 

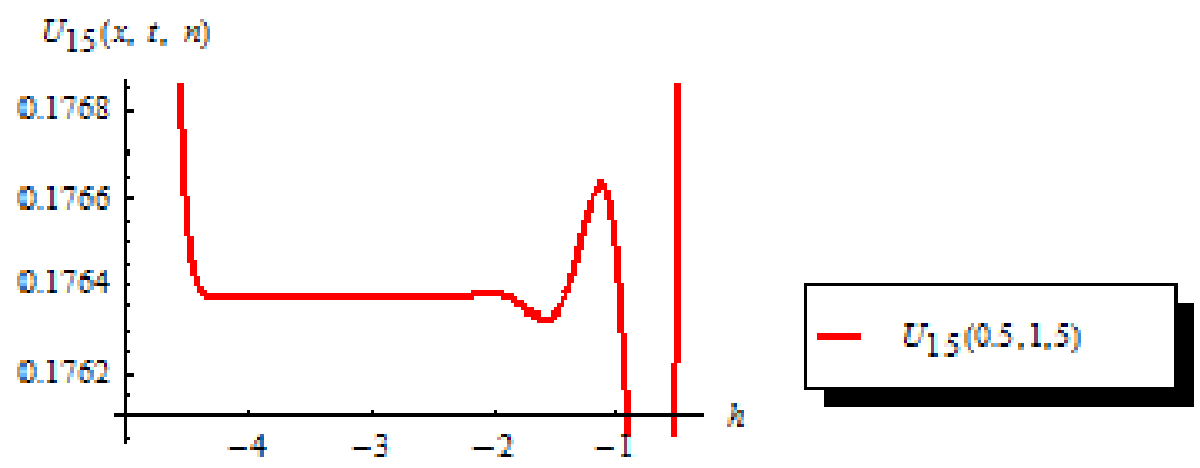

Fig. (2) $h$ - curve for the ( q-HAM; $n=5)$ approximation solution, $U_{15}(0.5,1 ; 5)$ of problem (11).

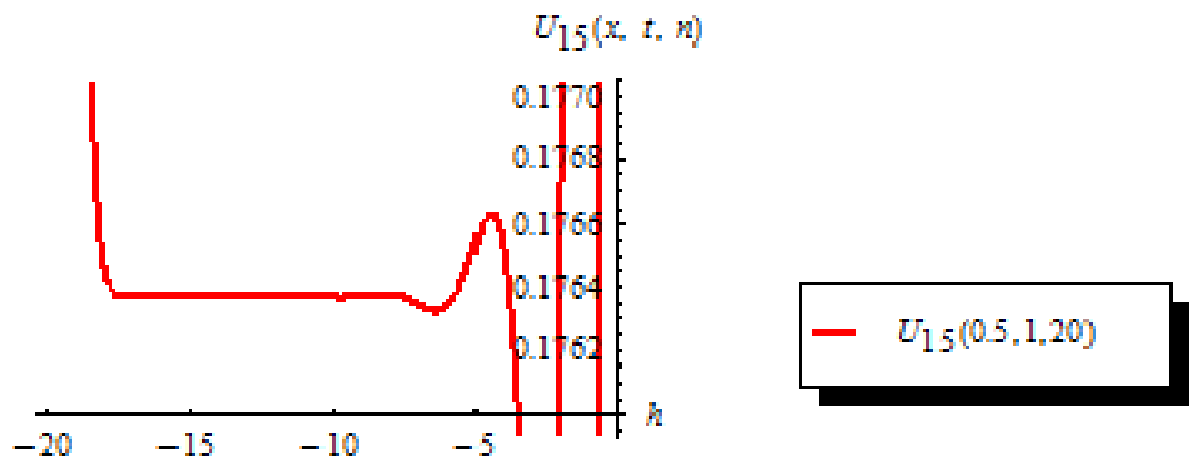

Fig. (3) $h$ - curve for the ( $\mathrm{q}-\mathrm{HAM} ; n=20)$ approximation solution, $U_{15}(0.5,1 ; 20)$ of problem (11).

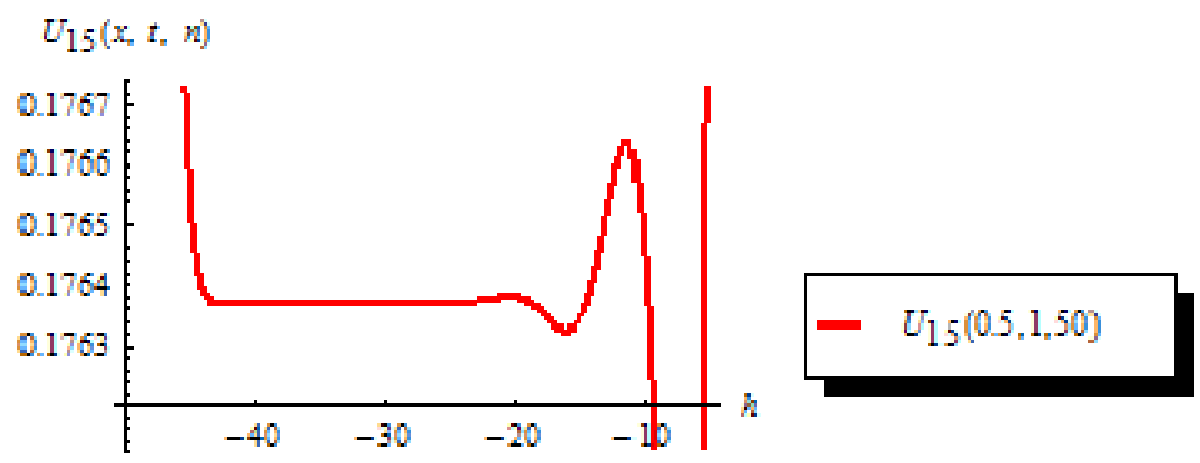

Fig. (4) $h$ - curve for the ( q-HAM; $n=50)$ approximation solution, $U_{15}(0.5,1 ; 50)$ of problem (11).
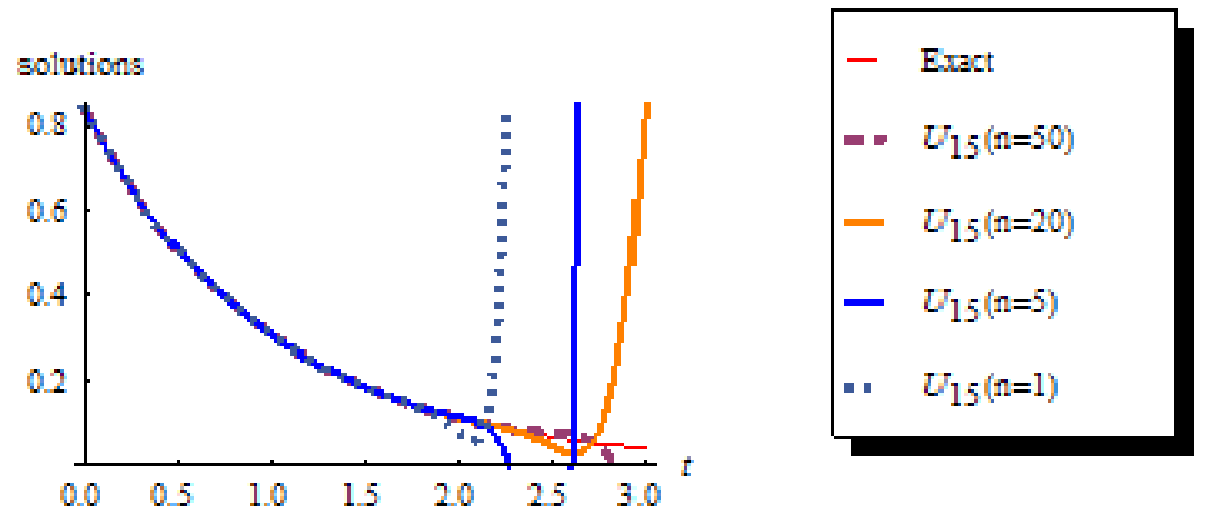

Fig. (5) Comparison between $U_{15}$ of HAM (q-HAM $(n=1)$ ) and q-HAM, $(n=5,20,50)$ with the exact solution of problem (11) at $x=1$ with, $(=-0.75, h=-3.55, h=-12.2, h=-28.11)$ respectively. 

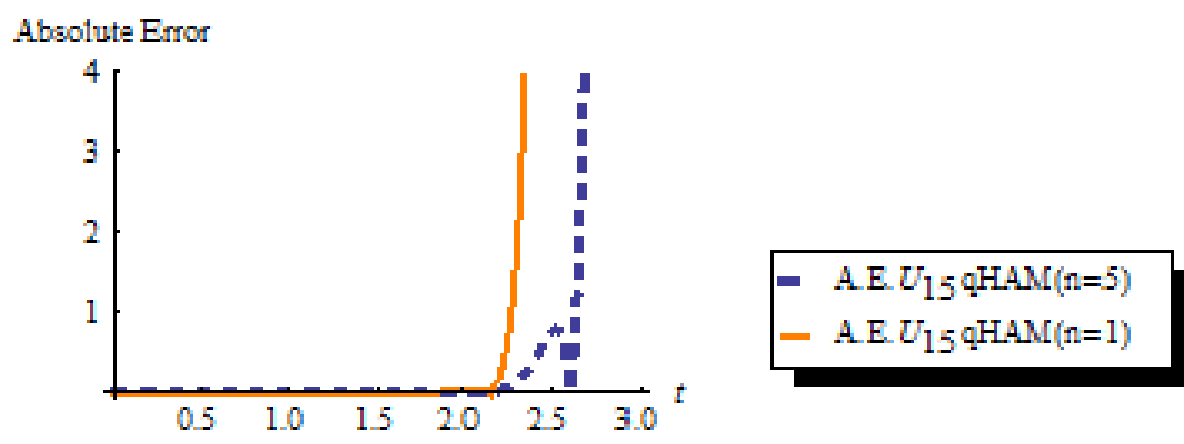

Fig. (6) The absolute errors of $U_{15}$ of q-HAM $(n=1, n=5)$ for problem (11), at $0 \leq t \leq 3$ and $x=1$ using $h=-0.75$ and $h=-3.55$.
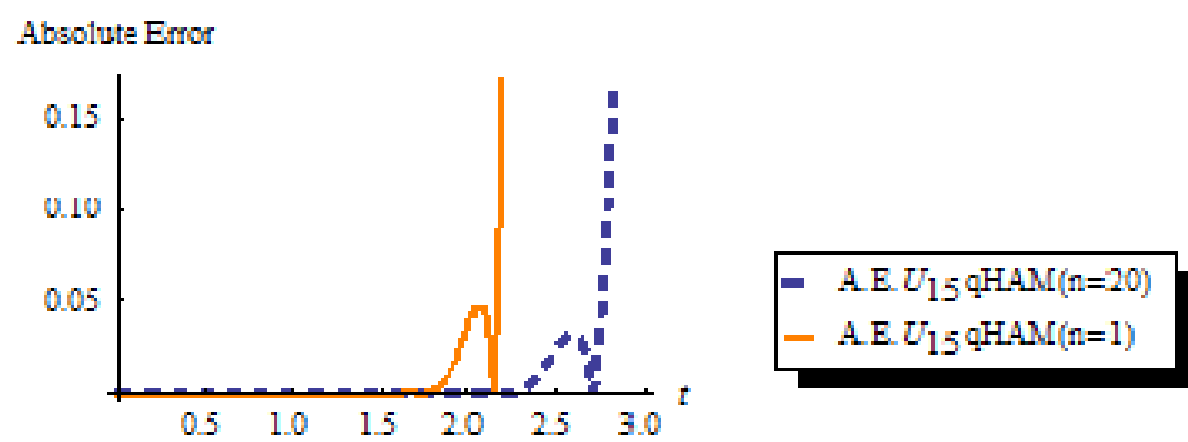

Fig. (7) The absolute errors of $U_{15}$ of q-HAM $(n=1, n=20)$ for problem (11), at $0 \leq t \leq 3$ and $x=1$ using $h=-0.75$ and $h=-12.2$.
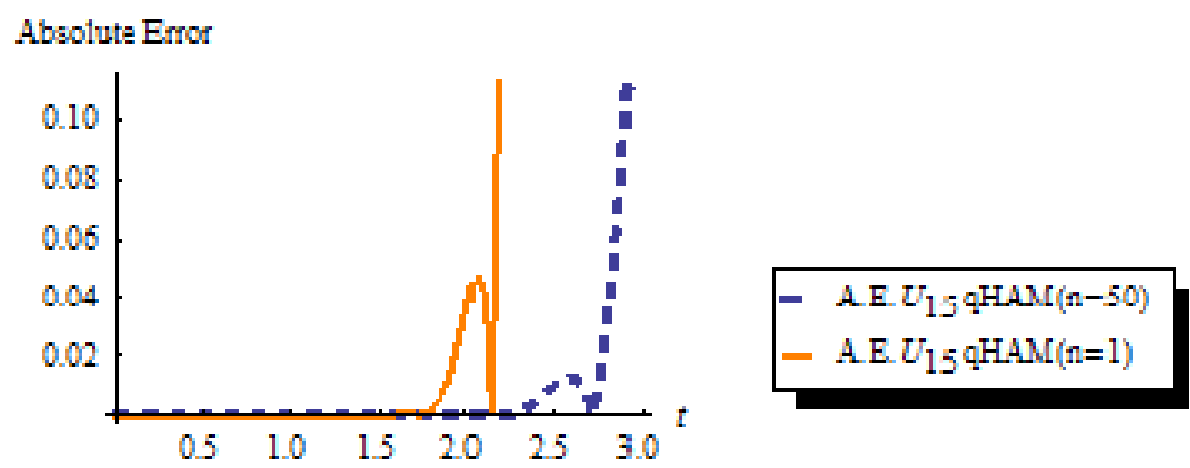

Fig. (8) The absolute errors of $U_{15}$ of q-HAM $(n=1, n=50)$ for problem (11), at $0 \leq t \leq 3$ and $x=1$ using $h=-0.75$ and $h=-28.11$.

$$
\begin{gathered}
u_{t t}+12 u_{t}+4 u=u_{x x}- \\
12 \sin (x) \sin (t)+4 \cos (\mathrm{t}) \sin (x)
\end{gathered}
$$

The initial conditions are given by

$$
\begin{gathered}
u(x, 0)=\sin (x), \\
u_{t}(x, 0)=0,0 \leq x \leq 4
\end{gathered}
$$

The exact solution by $[2,34]$ is

$$
u(x, t)=\cos (t) \sin (x)
$$

This problem was solved by HAM in [10]. For qHAM solution we choose the linear operator:

$$
L[\varnothing(x, t ; q)]=\frac{\partial^{2} \varnothing(x, t ; q)}{\partial t^{2}}
$$

with the property $L\left[c_{1}+c_{2} t\right]=0$, where $c_{1}, c_{2}$ are constants. Using initial approximation $u_{0}(x, t)=$ $\sin (x)$, we define a nonlinear operator as

$$
\begin{aligned}
N[\varnothing(x, t ; q)] & =\frac{\partial^{2} \emptyset(x, t ; q)}{\partial t^{2}}+12 \frac{\partial \phi(x, t ; q)}{\partial t} \\
+ & 4 \emptyset(x, t ; q)-\frac{\partial^{2} \emptyset(x, t ; q)}{\partial x^{2}}
\end{aligned}
$$


We construct the zero order deformation equation:

$$
\begin{gathered}
(1-n q) L\left[\varnothing(x, t ; q)-u_{0}(x, t)\right] \\
=q h N[\varnothing(x, t ; q)] .
\end{gathered}
$$

we can take $H(x, t)=1$, and the $m^{\text {th }}$ order deformation equation is :

$L\left[u_{m}(x, t)-k_{m} u_{m-1}(x, t)\right]=h R_{m}\left(\overrightarrow{u_{m-1}}(x, t)\right)(21)$ with the initial conditions for $m \geq 1$

$$
u_{m}(x, 0)=0
$$

Where $k_{m}$ as define by (10) and

$$
\begin{aligned}
& R_{m}\left(\overrightarrow{u_{m-1}}(x, t)\right)=\frac{\partial^{2} u_{m-1}(x, t)}{\partial t^{2}}+12 \frac{\partial u_{m-1}(x, t)}{\partial t} \\
&+4 u_{m-1}(x, t)-\frac{\partial^{2} u_{m-1}(x, t)}{\partial x^{2}}+ \\
&\left(1-\frac{1}{n} k_{m}\right)(12 \sin (x) \sin (t)-4 \cos (\mathrm{t}) \sin (x))
\end{aligned}
$$

Now the solution of equation (18) for $m \geq 1$ becomes

$$
\begin{aligned}
& u_{m}(x, t)=k_{m} u_{m-1}(x, t) \\
& +h \int_{0}^{t} \int_{0}^{s} R_{m}\left(\overrightarrow{u_{m-1}}(x, r)\right) d r d s+c_{1} \\
& +c_{2} t
\end{aligned}
$$

where the constant of integration $c_{1}$ and $c_{2}$ are determined by the initial conditions (22).

We can obtain components of the solution using qHAM as follows:

$$
\begin{aligned}
u_{1}(x, t)= & \frac{1}{2} h\left(-8+24 t+5 t^{2}+8 \operatorname{Cos}[t]\right. \\
& -24 \operatorname{Sin}[t]) \operatorname{Sin}[x] \\
u_{2}(x, t)= & \frac{1}{2} h n\left(-8+24 t+5 t^{2}+8 \operatorname{Cos}[t]\right. \\
& -24 \operatorname{Sin}[t]) \operatorname{Sin}[x]+\frac{1}{24} h^{2}(-3072 \\
& +t(-2304+t(1548+5 t(96 \\
& +5 t)))+3072 \operatorname{Cos}[t] \\
& +2304 \operatorname{Sin}[t]) \operatorname{Sin}[x] \\
u_{m}(x, t),(m & =3,4,5, \ldots) \quad \operatorname{can} \quad \text { be calculated }
\end{aligned}
$$

similarly. Then the series solution expression by qHAM can be written in the form:

$$
\begin{gathered}
u(x, t ; n ; h) \cong U_{M}(x, t ; n ; h)= \\
\sum_{i=0}^{M} u_{i}(x, t ; n ; h)\left(\frac{1}{n}\right)^{i}
\end{gathered}
$$

Equation (23) is an approximate solution to the problem (18) in terms of the convergence parameters $h$ and $n$. To find the valid region of $h$, the $h$-curves given by the $15^{\text {th }}$ order q-HAM approximation at $(x=0.5, t=1)$ and different values of $n$ are drawn in figures $(9-12)$. These figures show the interval of $h$ at which the value of $U_{15}(x, t ; n)$ is constant at certain values of , $t$ and $n$. We choose the horizontal line parallel to $x$ - axis $(h)$ as a valid region which provides us with a simple way to adjust and control the convergence region of the series solution (23). From these figures, the region of $h$ for the values of $x, t$ and $n$ in the curves becomes larger as $n$ increase. Figure (13) shows the comparison between $U_{15}$ of HAM and $U_{15}$ of q-HAM using different values of $n$ with the exact solution (19), which indicates that the speed of convergence for q-HAM with $n>1$ is faster in comparison with $n=1$. Figures $(14-16)$ show that the series solutions obtained by q-HAM at $n>1$ converge faster than $n=1$ (HAM).

\section{Conclusion}

In this paper, the one-dimensional hyperbolic telegraph equations are solved by employing the q-homotopy analysis method (q-HAM). The convergence of the q-HAM is numerically studied by comparison with the exact solutions of the problems. The results shows that the convergence of the q- homotopy analysis method is more accurate than the convergence of the homotopy analysis method (HAM). 

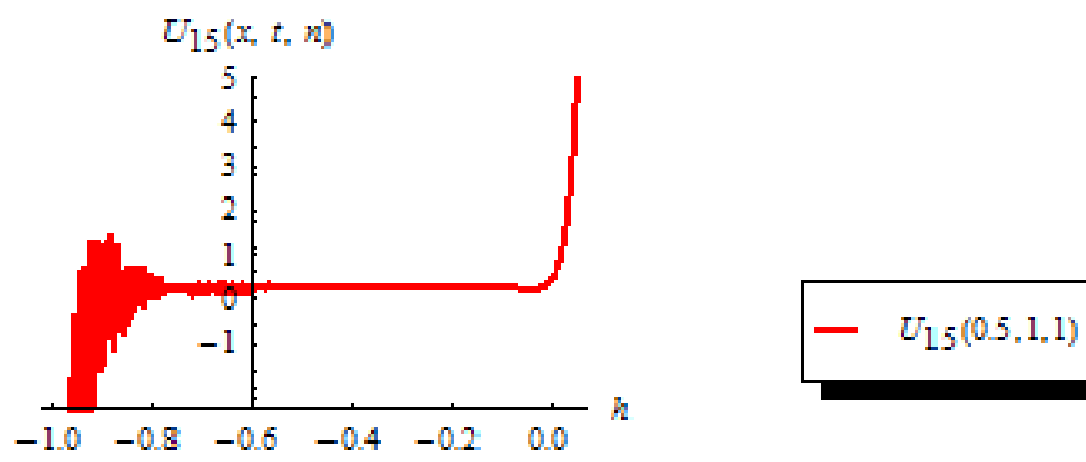

Fig. (9) $h$ - curve for the HAM (q-HAM; $n=1)$ approximation solution, $U_{15}(0.5,1 ; 1)$ of problem (18).
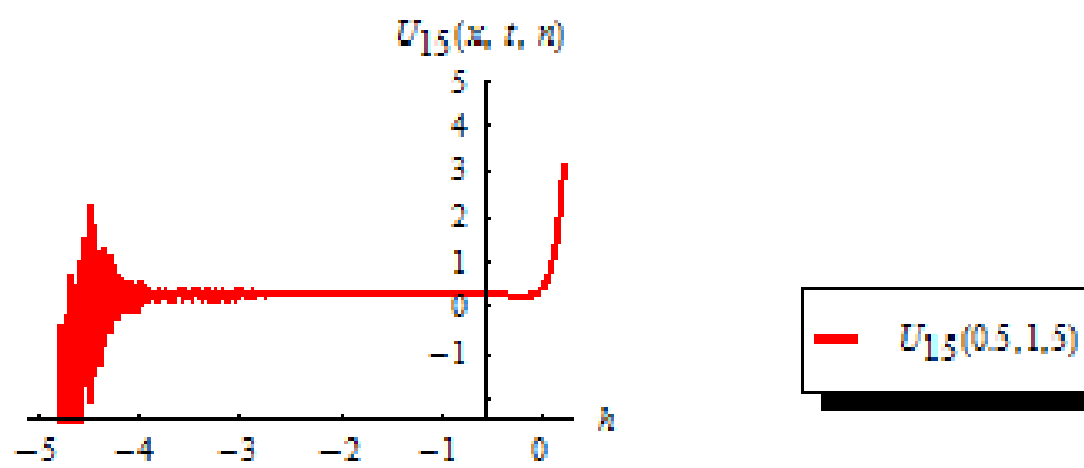

Fig. (10) $h$ - curve for the ( q-HAM; $n=5)$ approximation solution, $U_{15}(0.5,1 ; 5)$ of problem (18).
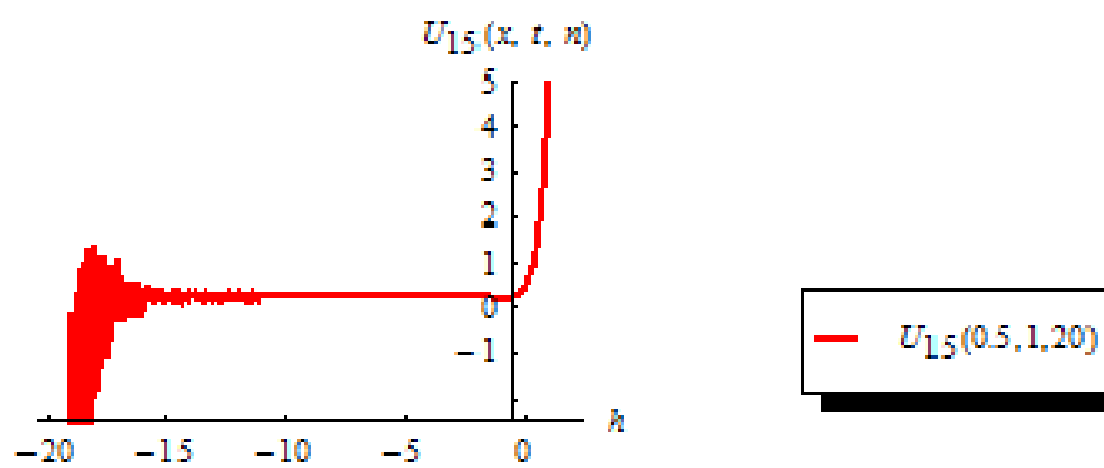

Fig. (11) $h$ - curve for the ( $q-H A M ; n=20$ ) approximation solution, $U_{15}(0.5,1 ; 5)$ of problem (18).
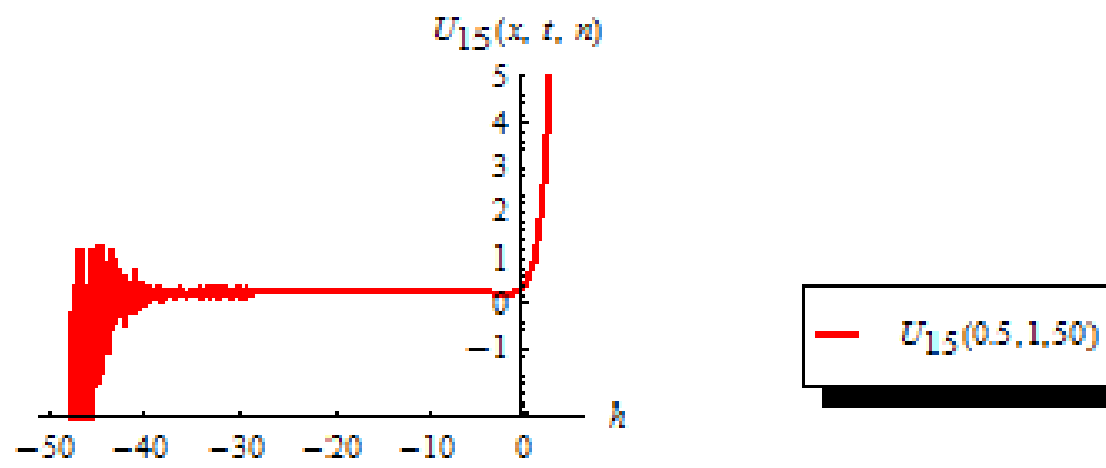

Fig. (12) $h$ - curve for the ( q-HAM; $n=50)$ approximation solution, $U_{15}(0.5,1 ; 5)$ of problem (18). 

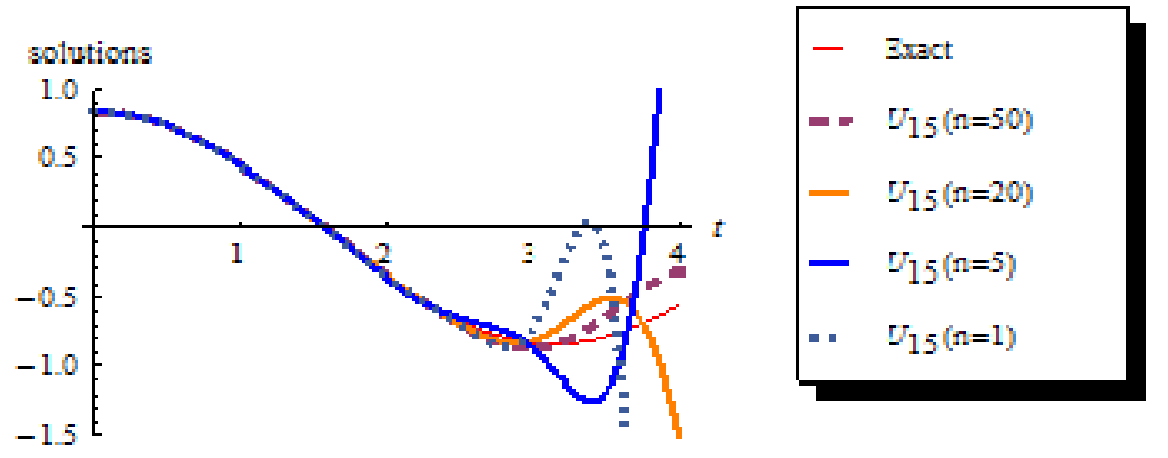

Fig. (13) Comparison between $U_{15}$ of HAM (q-HAM $(n=1)$ ) and q-HAM, $(n=5,20,50)$ with the exact solution of problem (18) at $x=1$ with, $(h=-0.35, h=-1.5, h=-5, h=-7.5)$ respectively.
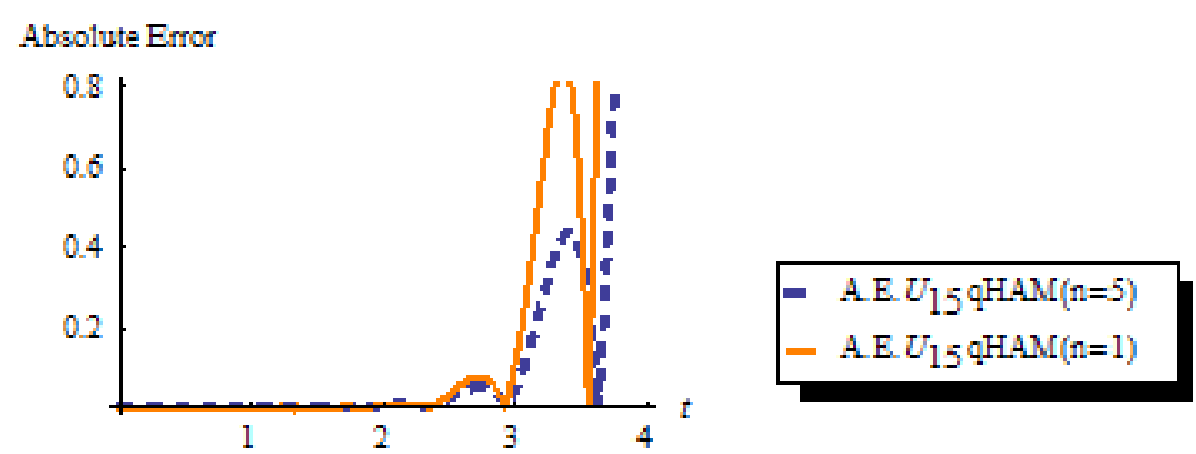

Fig. (14) The absolute errors of $U_{15}$ of q-HAM $(n=1, n=5)$ for problem (18), at $0 \leq t \leq 4$ and $x=1$ using $h=-0.35$ and $h=-1.5$.
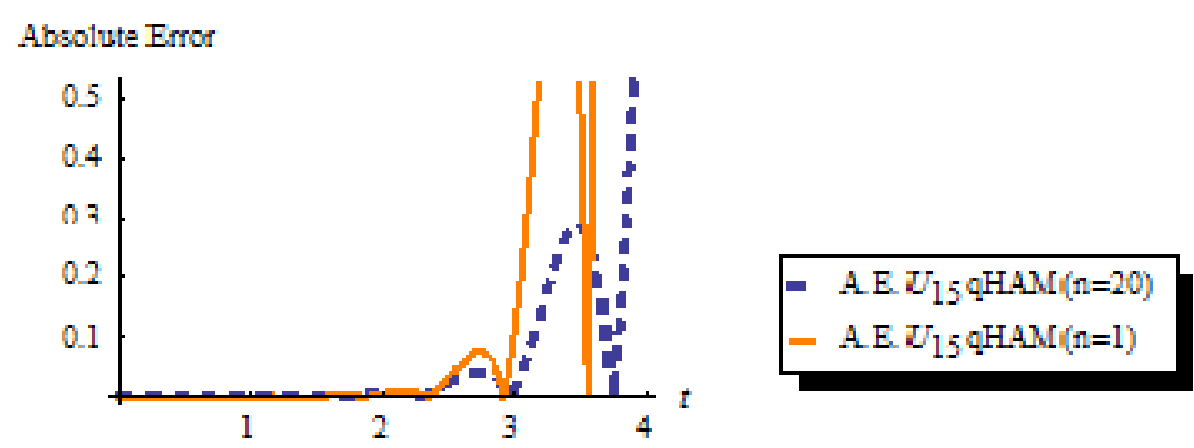

Fig. (15) The absolute errors of $U_{15}$ of q-HAM $(n=1, n=20)$ for problem (18), at $0 \leq t \leq 4$ and $x=1$ using $h=-0.35$ and $h=-5$.
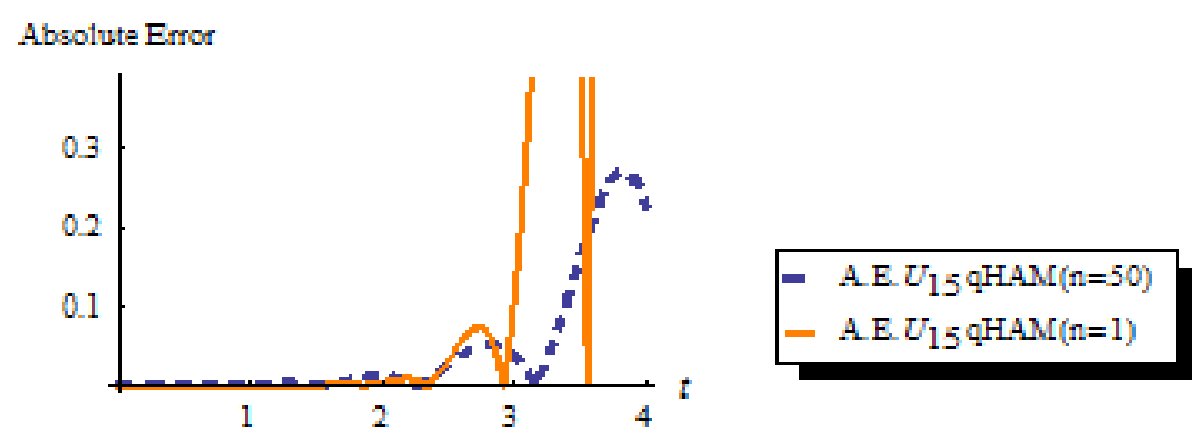

Fig. (16) The absolute errors of $U_{15}$ of q-HAM $(n=1, n=50)$ for problem, (18) at $0 \leq t \leq 4$ and $x=1$ using $h=-0.35$ and $h=-7.5$. 


\section{References}

[1] Bohme G., Non-Newtonian Fluid Mechanics, NewYork: North-Holland, 1987.

[2] Dehghan M. and Lakestani M., The use of Chebyshev cardinal functions for solution of the second-order one-dimensional telegraph equation. Numer. Methods Partial Diff. Eq., 25(2009) 931-938.

[3] Dehghan M. and Shokri A., A numerical method for solving the hyperbolic telegraph equation. Numer Methods Partial Diff. Eq., 24(2008) 1080-1093.

[4] Dehghan M. and Shokri A., A mesh less method for numerical solution of a linear hyperbolic equation with variable coefficients in two space dimensions. Numer Methods Partial Diff. Eq., 25(2009) 494-506.

[5] Dehghan M. and Mohebbi A., High order implicit collocation method for the solution of twodimensional linear hyperbolic equation. Numer Methods Partial Diff. Eq., 25(2009) 232-243.

[6] El-Tawil M. A. and Huseen S.N., The q-Homotopy Analysis Method (q-HAM), International Journal of Applied mathematics and mechanics, 8 (15): 51-75( 2012).

[7] El-Tawil M. A. and Huseen S.N., On Convergence of The q-Homotopy Analysis Method, Int. J. Contemp. Math. Sciences, Vol. 8 (2013) no. 10, 481 - 497.

[8] Evans D. J., Bulut H., Thenumerical solution of thetelegraph equation by the alternating

[9] group explicit method, Computer Mathematics, 80 (2003) 1289- 1297.

[10] Gao, F. and Chi C., Unconditionally stable difference schemes for a one-space-dimensional linear hyperbolic equation. Appl. Math. Comput., 187(2007) 1272-1276.

[11] Hosseini M.M., Syed Tauseef Mohyud-Din, Hosseini S.M. and Heydari M., Study on Hyperbolic Telegraph Equations by Using Homotopy Analysis Method, Studies in Nonlinear Sciences 1 (2): 50-56( 2010).

[12] Huseen, S. N. and Grace, S. R., Approximate Solutions of Nonlinear Partial Differential Equations by Modified q-Homotopy Analysis Method (mq-HAM), Hindawi Publishing Corporation, Journal of Applied Mathematics, Article ID 569674, 9 pages http:// dx.doi.org/10.1155/ 2013/ 569674.

[13] Huseen S. N., Grace S. R. and El-Tawil M. A., The Optimal q-Homotopy Analysis Method (Oq-HAM), International Journal of Computers \& Technology, Vol 11, No. 8. (2013)

[14] Huseen Shaheed N. Application of optimal q-homotopy analysis method to second order initial and boundary value problems. Int J Sci Innovative Math Res (IJSIMR) 2015;3(1):18-24.
[15] Huseen S. N., Solving the K(2,2) Equation by Means of the q-Homotopy Analysis Method (q-HAM), International Journal of Innovative Science, Engineering \& Technology, Vol. 2 Issue 8, August 2015.

[16] Huseen S. N., Series Solutions of Fractional Initial-Value Problems by q-Homotopy Analysis Method, International Journal of Innovative Science, Engineering \& Technology, Vol. 3 Issue 1, January 2016.

[17] Iyiola O. S., q-Homotopy Analysis Method and Application to Fingero-Imbibition phenomena in double phase flow through porous media, Asian Journal of Current Engineering and Maths 2(2013) 283 - 286.

[18] Jeffrey A., Advanced Engineering Mathematics. Harcourt Academic Press, 2002.

[19] Jeffrey A., Applied Partial Differential Equations. NewYork: Academic Press, 2002.

[20] Jordan P. M., Meyer M. R., Puri A., Causal implications of viscous damping in compressible fluid flows. Phys Rev, 62 (2000) 7918-7926.

[21] Liao S. J., Proposed homotopy analysis techniques for the solution of nonlinear problems, Ph.D. dissertation, Shanghai Jiao Tong University, Shanghai, 1992.

[22] Liao S. J., An approximate solution technique which does not depend upon small parameters: a special example, Int. J. Non-linear Mech. 30:371-380(1995).

[23] Liao S. J., An approximate solution technique which does not depend upon small parameters (Part 2): an application in fluid mechanics, Int. J. Non-linear Mech. 32:815-822(1997)

[24] Liao S. J., An explicit, totally analytic approximation of Blasius viscous flow problem, Int. J.Non-Linear Mech. 34:759-778(1999).

[25] Liao S. J., A uniformly valid analytic solution of 2D viscous flow past a semi-infinite flat plate. J. Fluid Mech. 385:101-128(1999).

[26] Liao S. J., Campo A., Analytic solutions of the temperature distribution in Blasius viscous flow problems, J.Fluid Mech. 453:411-425(2002).

[27] Liao S. J., An explicit analytic solution to the Thomas-Fermi equation, Appl. Math. Comput. 144: 495-506(2003).

[28] Liao S. J., On the analytic solution of magnetohydrodynamic flows of nonNewtonian fluids over a stretching sheet, J. Fluid Mech. 488:189-212(2003).

[29] Liao S. J., On the homotopy analysis method for nonlinear problems, Appl. Math. Comput.147: 499-513(2004) .

[30] Liao S. J., Magyari E., Exponentially decaying boundary layers as limiting cases of families of algebraically decaying ones, Z. Angew. Math. Phys. 57:777-792(2006). 
[31] Liao S. J., Tan Y., A general approach to obtain series solutions of nonlinear differential equations, Stud. Appl. Math. 119:297-354(2007).

[32] Liao S. J., A general approach to get series solution of non-similarity boundary-layer flows, Commun. Nonlinear Sci. Numer. Simulat. 14:2144-2159(2009).

[33] Liao S. J., Beyond perturbation: Introduction to the homotopy analysis method, CRC press LLC, Boca Raton, 2003.

[34] Mohanty R. K., New unconditionally stable difference schemes for the solution of multi-dimensional telegraphic equations, Computer Mathematics, 86 (2008) 2061 2071.

[35] Mohebbi A. and Dehghan M., High order compact solution of the one-space- dimensional linear hyperbolic equation. Numer Methods Partial Diff Eq., 24 (5) (2008) 1222-1235.

[36] Pascal H., Pressure wave propagation in a fluid flowing through a porous medium and problems related to interpretation of Stoneley's wave attenuation in acoustical well logging, Engineering Science, 24 (1986) 1553-1570.

[37] Pozar D. M., Microwave Engineering. NewYork: Addison-Wesley, 1990.

[38] Saadatmandi A. and Dehghan M., Numerical solution of hyperbolic telegraph equation using the Chebyshev tau method. Numer Methods Partial Diff. Eq., DOI $10.1002 /$ num. 